\title{
Patterning of circular structure arrays with interference lithography
}

\author{
Harun H. Solak ${ }^{\mathrm{a})}$ and Christian David \\ Laboratory for Micro- and Nanotechnology, Paul Scherrer Institute, 5232 Villigen PSI, Switzerland
}

(Received 30 June 2003; accepted 8 September 2003; published 9 December 2003)

\begin{abstract}
We have developed an interference lithography method that enables the production of circular periodic patterns. The range of accessible patterns includes concentric circular rings, spiral- and spoke-like patterns, and dots positioned periodically on concentric circular tracks. The method is based on the use of transmission diffraction gratings to create circular beams with wave fronts that are not accessible with reflective or refractive optics. Diffraction gratings with equally spaced concentric circles are used to obtain diverging or converging conical waves with radial phase variation and spiral-like gratings are used to obtain waves with the phase varying in both radial and azimuthal directions. We have experimentally demonstrated the method with electron-beam written diffraction gratings, which we illuminated with a coherent laser beam to form the interference patterns. Many different designs are possible that bring two, three, or more beams together to form a desired interference pattern. The technique should in general be useful in applications requiring circular periodic patterns, which may include production of patterned magnetic media, spoke patterns for angular encoders, and photonic crystals with circular symmetry. (c) 2003 American Vacuum Society. [DOI: 10.1116/1.1622943]
\end{abstract}

\section{INTRODUCTION}

Periodic patterns with circular symmetry are needed in many applications. For example, in patterned magnetic media applications it is desirable to have individual magnetic bit elements periodically positioned on concentric circular tracks. ${ }^{1}$ This circular arrangement facilitates addressing of individual bits on a spinning disk by the read/write head. Angular encoders are another example for the use of circular periodic structures in which spoke-like radial patterns are used to measure the angular position of rotary devices. Other potential applications for circular periodic patterns include ring-shaped photonic band gap crystals.

Interference lithography (IL) is well suited for patterning large areas with high density periodic structures. Onedimensional line/space patterns are readily obtained by the interference of two mutually coherent beams. Multiexposure or multibeam schemes have been developed for making twodimensional arrays. ${ }^{2}$ Two-dimensional arrays obtained in this way normally have the symmetry of a rectangular or hexagonal grid. More complicated holographic techniques have been used to make circular structures such as Fresnel zone plates $^{3}$ and concentric circular ring patterns with constant periods. ${ }^{4}$ Refractive lenses with high precision and quality are needed in this holographic method to obtain the wave fronts. Moreover, the periodicity of the patterns produced is limited to the radial direction since it would be difficult if not impossible to produce a wave with continuous phase variation along the azimuthal direction using refractive optics.

Interference patterns obtained using transmission diffraction gratings have been recognized for their number of advantageous properties. ${ }^{5}$ For example, imaging of one grating by a second one lets one build interferometers that do not require either spatial or temporal coherence. ${ }^{6}$ In multibeam

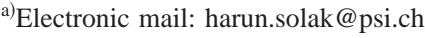

interference applications gratings can be used to satisfy the required phase relations, which is an extremely demanding task in implementations with reflective optics. ${ }^{7}$ In this article we report the development of an IL method based on diffraction gratings to extend the range of patterns accessible with interference lithography to include patterns with circular symmetry. In this method, transmission diffraction gratings are used to obtain light waves that interfere and form circularly symmetric intensity patterns. Gratings with concentric circular rings are used to obtain diverging or converging waves with radial phase variation and gratings in the shape of spirals or spoke patterns are used to obtain waves with the phase varying in both radial and azimuthal directions. Many variations of the scheme, an example of which is shown in Fig. 1 are possible. We have designed and tested a number of them experimentally using e-beam written diffraction gratings.

\section{DESIGN OF THE DIFFRACTION GRATINGS}

Many different types of circular periodic structures can be obtained by interference of beams that are diffracted by gratings. Therefore, rather than trying to cover all the possible variations we will describe the principles involved in designing such an interference scheme. The range of possible circular patterns includes one-dimensional patterns such as concentric circular rings (tracks), spoke- or spiral-like patterns, and two-dimensional patterns that are combinations of the one-dimensional patterns listed above. For example, a combination of a circular track pattern with a spoke pattern yields a pattern of periodic dots arranged on circular tracks. In general, one-dimensional patterns are obtained by two interfering beams. The "combination" mentioned above of one-dimensional patterns to obtain two-dimensional patterns can be realized by either multibeam or multiple exposure techniques. In the former, three or more coherent beams created by diffraction gratings written on a single substrate in- 


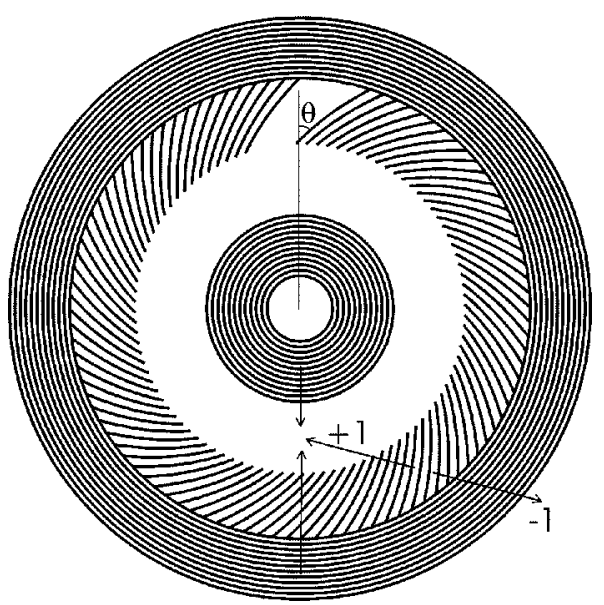

FIG. 1. Schematic layout of a grating configuration for creating interference patterns with circular symmetry. Diffracted beams from the three distinct grating regions coincide in the clear annular region, indicated by the arrows, and form a two-dimensional pattern with intensity peaks periodically positioned along circular tracks. Both the ingoing (positive) and outgoing (negative) orders from the spiral grating are shown.

terfere and create the pattern. In the multiple exposure route, two one-dimensional (two-beam) exposures are performed consecutively to obtain the desired two-dimensional dose distribution in the photoresist.

We use two main types of diffraction grating to form the circular patterns. First, gratings consisting of equally spaced concentric rings are used to create diffracted beams with conical wave fronts, i.e., with linearly varying phase in the radial direction given by

$$
\beta=2 \pi n r / p,
$$

where $n$ is the diffraction order, $r$ is the radial coordinate, and $p$ is the period of the diffraction grating. Second, spiral-like gratings are used to create beams with phase varying in both the radial and azimuthal directions. The spoke pattern can be considered to be a special case of this "spiral" type, when the lines of the grating lie in the radial direction. The phase variation in the azimuthal direction is given by

$$
\beta=2 \pi N n \phi,
$$

where $N$ is the number of periods in the spiral grating and $\phi$ is the azimuthal angle. Note that the phase variation in the azimuthal direction is independent of both the angle of the spiral, $\theta$, and the, radius $r$. We should note that in our designs and experimental work we used only equiangular spirals, even though other spiral types are not excluded.

In designing the interference masks we use a geometrical approach that requires proper overlapping of the interfering beams and obtaining the desired periods in the intensity pattern. In this approach we assume that the incident beam is a normally incident plane wave and we use the well known grating equation locally to calculate where the diffracted beams go. In this approach we ignore the diffraction effects due to the finite aperture (extent) of the gratings. In reality, the diffracted beams undergo Fresnel diffraction as they travel from the grating to the sample plane and diffraction

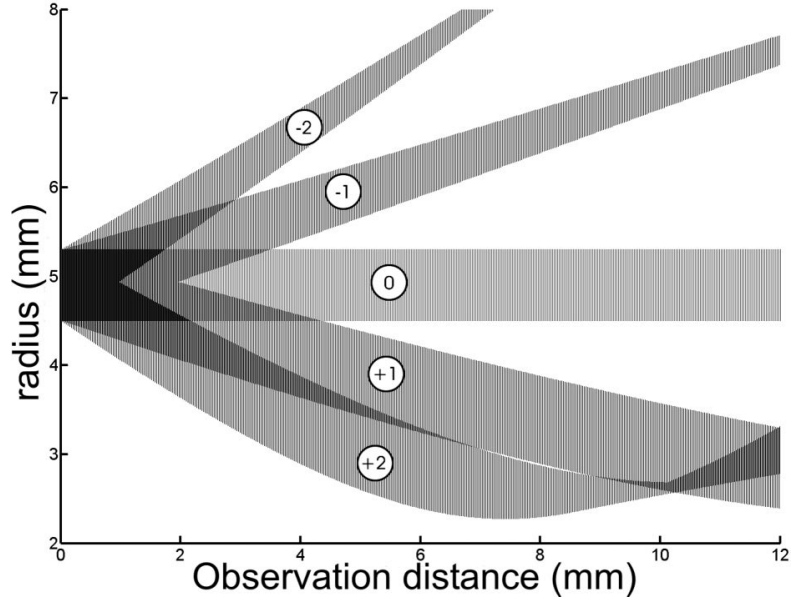

FIG. 2. Trajectories of several diffraction orders from a spiral grating as a function of the distance from the grating. The grating occupies the annular region between 4.5 and $5.3 \mathrm{~mm}$, where all the diffracted beams originate.

fringes are observed near the edges of the diffracted beam. Besides diffraction effects, the intensity of the diffracted beams varies in the radial direction due to the focusing effects of both the spiral and concentric type gratings. Figure 2 shows the calculated radial extent of some of the diffracted orders as a function of the distance from a spiral grating. We can make some useful observations regarding the behavior of the beams using this sample calculation. All the orders start out at the spiral grating which covers an annular range between 4.5 and $5.3 \mathrm{~mm}$. As the diffracted beams travel away from the grating, the radial range that they span changes. For example, the +2 order first converges towards smaller radius then turns around and starts diverging after being focused to a tight annular ring about $10 \mathrm{~mm}$ from the grating. We are usually interested in getting only one desired order (usually +1 or -1 ) in the radial range to be patterned. For example, in this case the +1 order is completely separated from the other orders at about $8 \mathrm{~mm}$ from the grating. Beams from other gratings of either spiral or circular type can interfere with this +1 order in this radial range and form an interference pattern. The intensity of light going into each order is determined by the duty cycle and the optical response of the grating bars. It is possible to strengthen some orders and reduce or even eliminate others by proper design of the gratings. The trajectory of the diffracted beams from a spiral grating, such as the ones shown in Fig. 2, depend on the parameters of the grating design such as the radial range it covers, the number of cycles in the grating, and the angle of the spiral with respect to the radial direction. In general it may not be possible to bring a beam originating from a spiral grating to a desired annular region with the properties required such as the azimuthal phase variation and order purity.

The spoke pattern, which is a special case of the spiral grating, has useful diffracting properties. The positive and negative diffraction orders originating from the spoke pattern are symmetric as shown in Fig. 3. These symmetric orders overlap completely at all distances from the grating and they never converge toward smaller radius regions. This makes 


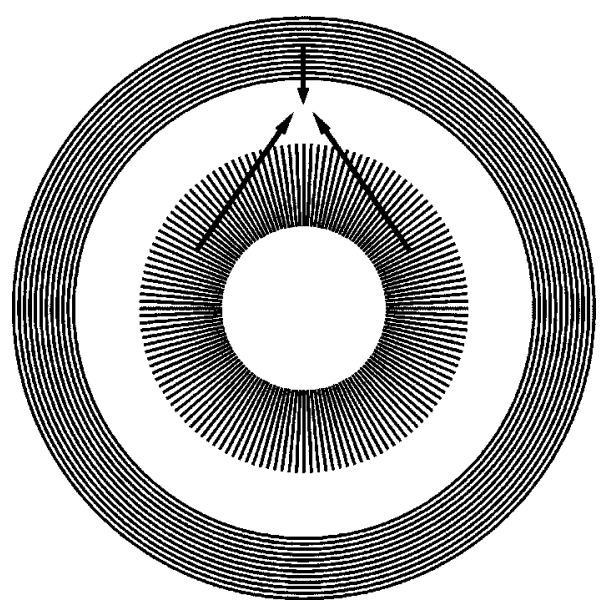

FIG. 3. Diagram showing the use of a spoke pattern as a diffraction grating. When the spoke pattern is used without the concentric circular grating on the outside, the two symmetric beams originating from the spoke form another spoke pattern with twice the number of lines. When all three beams, indicated by arrows, come together periodic intensity peaks along circular tracks are obtained.

the separation of diffraction orders (other than the symmetric ones) less complicated. The second advantage is that interference of two symmetric order beams is obtained automatically due to their self-alignment. Therefore one can use a single spoke pattern to obtain radial two-beam interference fringes which form another spoke pattern, with twice the number of lines. Another possibility is to use one spoke pattern in combination with a circular grating to form a threebeam interference pattern as seen in Fig. 3 .

\section{EXPERIMENT}

The gratings designed according to the principles described in Sec. II were written on quartz mask blanks by e-beam lithography. The mask patterns were written in a PMMA film and later transferred to the underlying $\mathrm{Cr}$ film via reactive ion etching. In this way pure amplitude type gratings were obtained since the Cr film almost completely absorbed light at the wavelength used. It is possible to etch the quartz films and remove the $\mathrm{Cr}$ films later to obtain phase gratings for better diffraction efficiency and suppression of certain orders, but that was not attempted in this study. We used continuous path control mode of the low energy e-beam exposure machine LION LV1 (Leica Microsystems, Jena, Germany) to write the circular and spiral structures. Several masks designed to obtain two- or three-beam interference were written.

Sample Si wafers were first coated with a $205 \mathrm{~nm}$ thick antireflective coating of XL20 type from Brewer Science (Rolla, MO). The antireflective coating was baked at $170{ }^{\circ} \mathrm{C}$ for $1 \mathrm{~min}$ to make it insoluble during resist development. Shipley 1805 photoresist was then spun on top to a thickness of $250 \mathrm{~nm}$. The IL exposures were done with light from a $\mathrm{He}-\mathrm{Cd}$ laser operating at $441.6 \mathrm{~nm}$. The light was filtered by a pinhole spatial filter to obtain spatially coherent and uniform illumination of the interference mask.

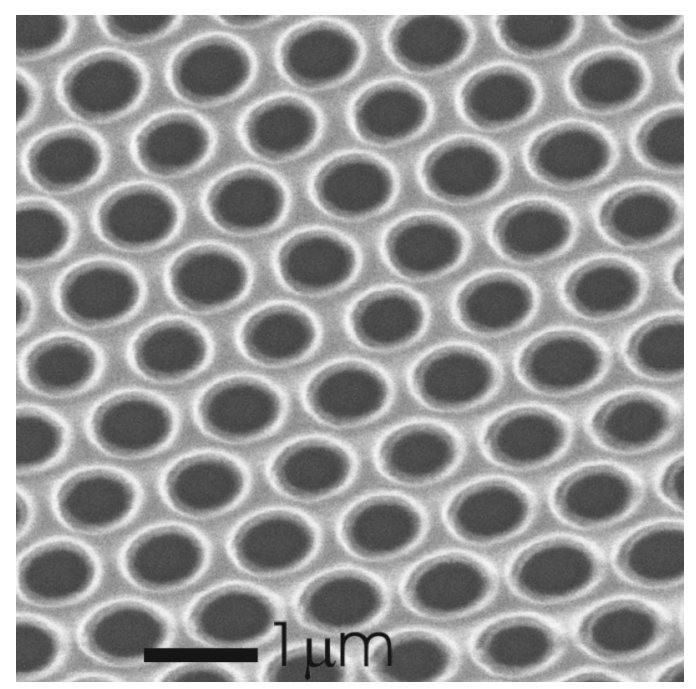

FIG. 4. Scanning electron micrograph of an array of holes obtained with the grating configuration shown in Fig. 1. The holes are positioned along circular tracks. The pattern period is $0.75 \mu \mathrm{m}$ in the radial direction and $1.2 \mu \mathrm{m}$ along the tracks.

\section{RESULTS AND DISCUSSION}

Figures 4 and 5 show two examples of patterns in photoresist obtained by circular interference exposures. Both samples were exposed with masks similar to the one shown in Fig. 1. The interference pattern was formed by three beams, two originating from the circular gratings and one originating from the spiral grating. The holes, which correspond to intensity peaks in the interference pattern, are positioned along equally spaced circular tracks. The number of holes around the circle is constant and is equal to the number of periods in the spiral gratings. Therefore the pattern period in the azimuthal direction changes with radius. The pattern in Fig. 4 covered an annular range of 5.0-6.5 $\mathrm{mm}$ and the one in Fig. 5 covered a range of $3.5-4.5 \mathrm{~mm}$. The arrangement of holes in neighboring tracks follows equiangular spiral

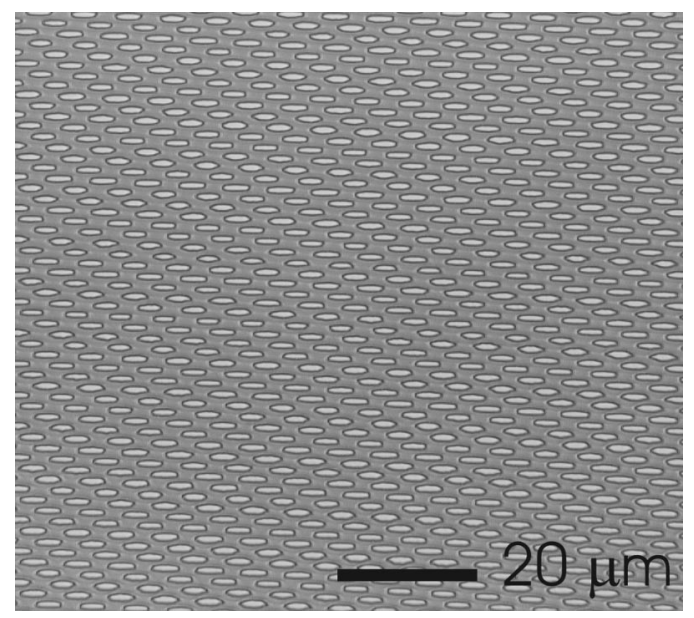

FIG. 5. Optical micrograph of an array of holes obtained with the grating configuration shown in Fig. 1. The holes are positioned along circular tracks. The pattern period is $1.5 \mu \mathrm{m}$ in the radial direction and $6.5 \mu \mathrm{m}$ along the tracks. 

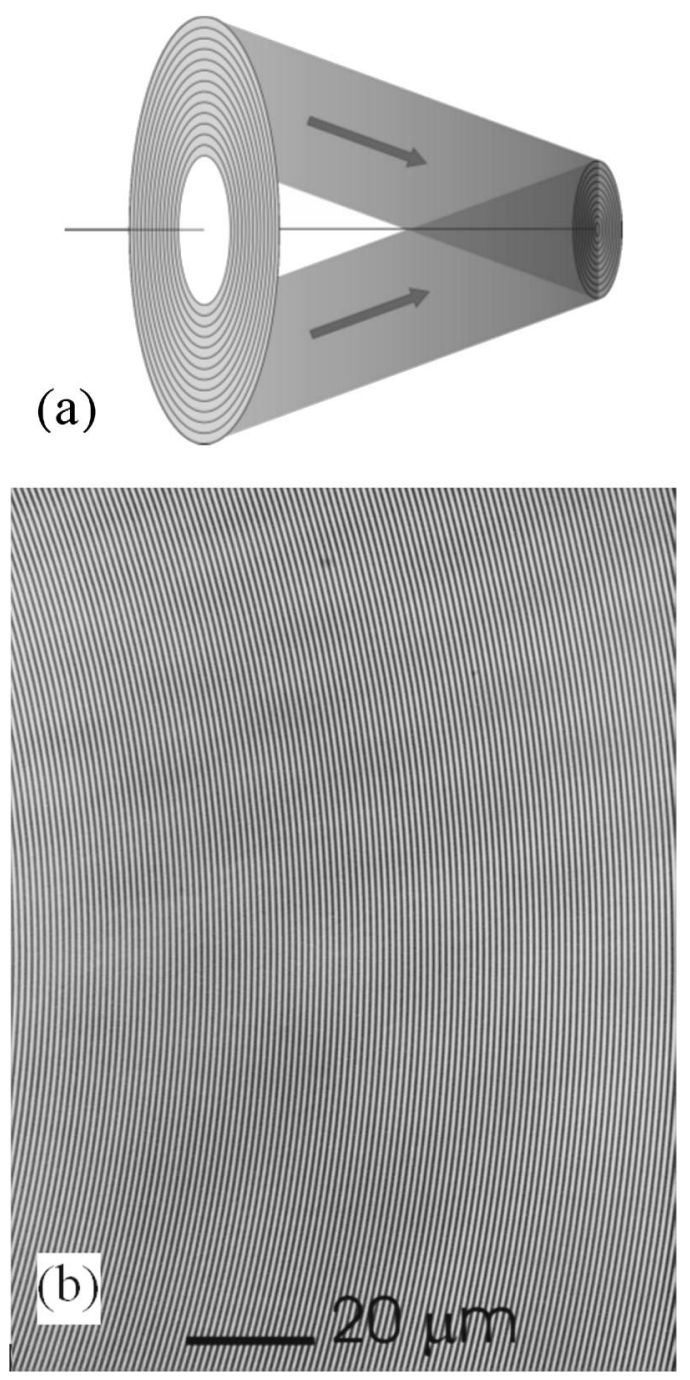

FIG. 6. (a) Diffracted light from a single circular diffraction grating interferes with itself and forms another circular grating with twice the spatial frequency. The diameter of the printed grating is equal to the diameter of the opaque area in the middle of the diffraction grating, which is also equal to the width of the diffraction grating. (b) Optical micrograph of a circular grating printed using the method in (a). The diffraction grating and the printed grating periods are 2 and $1 \mu \mathrm{m}$, respectively.

paths with the spiral angle being equal to the one written in the mask grating. Control of the hole positions in neighboring tracks may be important in patterned magnetic media applications where a certain arrangement may be preferred for magnetic coupling reasons or in circular photonic crystals where the location of the holes needs to have the symmetry of the desired photonic crystal.

In another experiment we used interference of a single diffraction order with itself from a circular grating as shown in Fig. 6 to create a circular grating pattern. The printed circular grating period is equal to half of the e-beam written diffraction grating. With this method the circular pattern is not limited to an annular region, i.e., it starts from zero radius. Assuming that the diffraction grating has uniform diffraction efficiency over its radial range, the light intensity in the exposed circular region will change with distance from the center. Figure 7 shows a calculated fringe pattern as a

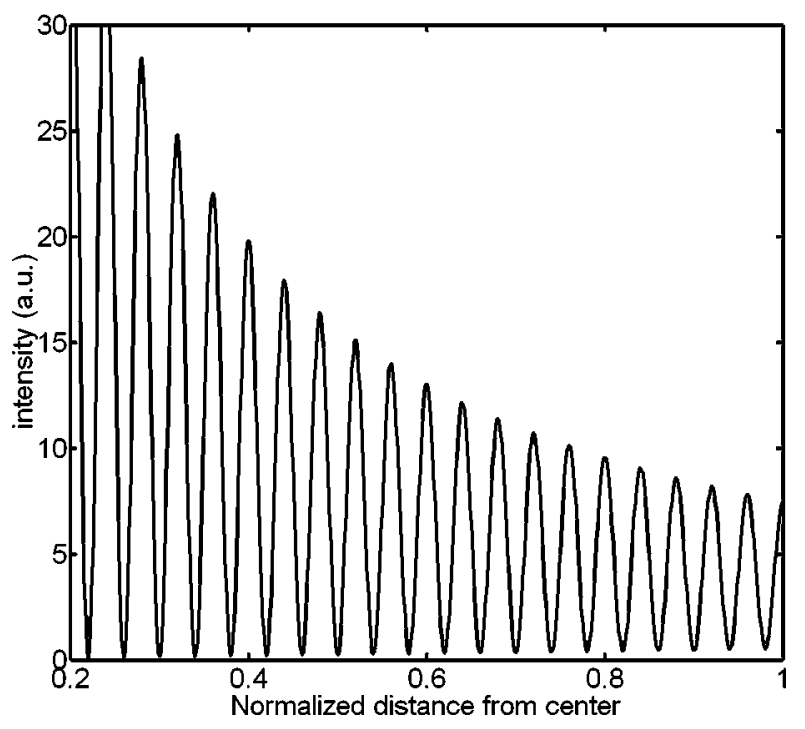

FIG. 7. Calculated interference pattern for the configuration in Fig. 6. The intensity peak varies approximately as $1 / r$, while the fringe modulation stays close to unity over the entire radial range. The radial distance is normalized to the outer radius of the interference pattern.

function of the radius. While the peak intensity changes significantly, the minimum intensity stays close to zero and the fringe modulation remains above $86 \%$ over the entire radial range. The duty cycle of the diffraction grating can be varied as a function of the radius to control the diffracted beam intensities and reduce the variation in intensity.

\section{CONCLUSIONS}

The use of gratings in IL enables the creation of periodic circular structures. The main advantages of IL are retained, including the large depth of focus and working distance, simplicity of the setup, and high resolution. In this initial work, we concentrated on demonstrating the concept rather than pushing the performance in terms of spatial resolution even though a $750 \mathrm{~nm}$ circular track period was readily achieved. We have demonstrated the creation of circular concentric rings and periodic arrays of dots on circular tracks. It is possible to form other kinds of patterns, including spiral- or spoke-like patterns, by combining beams from diffraction gratings in different ways.

The technique has potential application in making of patterned magnetic media in which the magnetic bits need to be positioned on circular tracks. In order to achieve the high resolution necessary in this potential application, synchrotron sources with wavelength in the EUV range or immersion lithography with laser sources may need to be used. Other potential applications include radial patterns for angular encoders and photonic crystals with circular symmetry.

${ }^{1}$ C. A. Ross, H. I. Smith, T. Savas, M. Schattenburg, M. Farhoud, M. Hwang, M. Walsh, M. C. Abraham, and R. J. Ram, J. Vac. Sci. Technol. B 17, 3168 (1999).

${ }^{2}$ X. Chen, S. H. Zaidi, S. R. Brueck, and D. J. Devine, J. Vac. Sci. Technol. B 14, 3339 (1996). 
${ }^{3}$ G. Schmahl, D. Rudolph, P. Guttmann, and O. Christ, in X-ray Microscopy, edited by G. Schmahl and D. Rudolph, Springer Series in Optical Science Vol. 43 (Springer, Heidelberg, 1984), pp. 63-74.

${ }^{4}$ S. Nishiwaki, J. Asada, K. Ohshima, and T. Kitagawa, Appl. Opt. 34, 7372 (1995)
${ }^{5}$ B. J. Chang, R. Alferness, and E. N. Leith, Appl. Opt. 14, 1592 (1975).

${ }^{6}$ A. Yen, E. H. Anderson, R. A. Ghanbari, M. L. Schattenburg, and H. I. Smith, Appl. Opt. 31, 4540 (1992).

${ }^{7}$ H. H. Solak, C. David, J. Gobrecht, L. Wang, and F. Cerrina, J. Vac. Sci. Technol. B 20, 2844 (2002). 